\title{
Computation of the General Relativistic Perihelion Precession and of Light Deflection via the Laplace-Adomian Decomposition Method
}

\author{
Man Kwong Mak, ${ }^{1}$ Chun Sing Leung, ${ }^{2}$ and Tiberiu Harko $\mathbb{D}^{3,4,5}$ \\ ${ }^{1}$ Departamento de Física, Facultad de Ciencias Naturales, Universidad de Atacama, Copayapu 485, Copiapó, Chile \\ ${ }^{2}$ Department of Applied Mathematics, Polytechnic University of Hong Kong, Hong Kong \\ ${ }^{3}$ Department of Physics, Babes-Bolyai University, Kogalniceanu Street, Cluj-Napoca 400084, Romania \\ ${ }^{4}$ School of Physics, Sun Yat-Sen University, Guangzhou 510275, China \\ ${ }^{5}$ Department of Mathematics, University College London, Gower Street, London WC1E 6BT, UK
}

Correspondence should be addressed to Tiberiu Harko; t.harko@ucl.ac.uk

Received 5 March 2018; Accepted 12 May 2018; Published 28 June 2018

Academic Editor: Luis Herrera

Copyright (C) 2018 Man Kwong Mak et al. This is an open access article distributed under the Creative Commons Attribution License, which permits unrestricted use, distribution, and reproduction in any medium, provided the original work is properly cited. The publication of this article was funded by SCOAP ${ }^{3}$.

We study the equations of motion of the massive and massless particles in the Schwarzschild geometry of general relativity by using the Laplace-Adomian Decomposition Method, which proved to be extremely successful in obtaining series solutions to a wide range of strongly nonlinear differential and integral equations. After introducing a general formalism for the derivation of the equations of motion in arbitrary spherically symmetric static geometries and of the general mathematical formalism of the Laplace-Adomian Decomposition Method, we obtain the series solution of the geodesics equation in the Schwarzschild geometry. The truncated series solution, containing only five terms, can reproduce the exact numerical solution with a high precision. In the first order of approximation we reobtain the standard expression for the perihelion precession. We study in detail the bending angle of light by compact objects in several orders of approximation. The extension of this approach to more general geometries than the Schwarzschild one is also briefly discussed.

\section{Introduction}

General relativity is a very successful theory of the gravitational field, whose predictions are in excellent agreement with a large number of astronomical observations and experiments performed at the scale of the Solar System. In particular, three fundamental tests of general relativity, the perihelion precession of planet Mercury [1,2], the bending of light by the Sun $[3,4]$, and the radar echo delay experiment $[5,6]$ have all fully confirmed, within the range of observational/experimental errors, the predictions of Einstein's theory of gravity. But the importance of these effects goes far beyond the limits of the Solar System. A fast full general relativistic method to simultaneously constrain the mass of massive black holes, their spin, and the spin direction by considering both the motion of a star and the propagation of photons from the star to a distant observer was developed in [7]. The spin-induced effects on the projected trajectory and redshift curve of a star depend on both the value and the direction of the spin. The maximum effects over a full orbit can differ by a factor up to more than one order of magnitude for cases with significantly different spin directions. In [8] it was shown that the spin of the massive black hole at the Galactic Center can be constrained with $1 \sigma$ error $<\sim 0.1$ or even greater than 0.02 by monitoring the orbital motion of a star with semi major axis $<\sim 300$ AU and eccentricity $>\sim 0.95$ over a period shorter than a decade through future facilities. An improvement in astrometric precision would be more effective at improving the quality of constraining the spin than an improvement in velocity precision. Shortperiod stars orbiting around the supermassive black hole in our Galactic Center can successfully be used to probe the 
gravitational theory in a strong regime [9]. By using 19 years of observations of the two best measured short-period stars orbiting our Galactic Center constraints on a hypothetical fifth force that arises in some extended theories of gravity or in some models of dark matter and dark energy were obtained in [9]. No deviations from general relativity were found, and the fifth force strength was restricted to an upper 95\% confidence limit of $|\alpha|<0.016$ at a length scale of $\lambda=150$ astronomical units. Moreover, 95\% confidence upper limit on a linear drift of the argument of periastron of the short-period star S0-2 was obtained, a result that opens the possibility of testing gravitational theories using orbital dynamic in the strong gravitational regime of a supermassive black hole. The S-star cluster in the Galactic Center allows the study of physics close to a supermassive black hole, including distinctive dynamical tests of general relativity [10], where a new and practical method for the investigation of the relativistic orbits of stars in the gravitational field near Sgr A* was developed, by using a first-order post-Newtonian approximation to calculate the stellar orbits with a broad range of periapse distance $r_{p}$. For in depth discussions of the experimental and Solar System tests of general relativity, see [11] and [12], respectively.

Due to its importance in many applications, the study of the motion of massive or massless particles in different geometries obtained as solutions of Einstein's gravitational field equations and of their extensions is a fundamental field of general relativity. The first exact solution of the vacuum field equations was the Schwarzschild solution [13], which can be used efficiently to explain all astronomical observations at the scale of the Solar System. The exact equation of motion in Schwarzschild geometry is highly nonlinear, and therefore to obtain the observable physical parameters approximate methods must be used. The first-order approximation of the equation of motion already gives the correct approximation of the perihelion precession of Mercury and of the deflection of light by the Sun [11]. However, due to the importance of the problem many mathematical techniques for the study of the astrometric properties of the planetary motions and of the light have been developed. A standard approach is based on the solution of the Hamilton-Jacobi equation [13]

$$
g^{\mu \nu} \frac{\partial S}{\partial x^{\mu}} \frac{\partial S}{\partial x^{\nu}}-m^{2} c^{2}=0,
$$

where $g^{\mu \nu}$ are the components of the metric tensor and $m$ is the mass of the particle. By representing $S$ as $S=-E t+M \varphi+$ $S_{r}(r)$, where $E$ and $M$ are the constants of the energy and angular momentum, one can obtain the full solution of the equation of motion in Schwarzschild geometry in an integral form as [13]

$$
\begin{aligned}
c t & =\frac{E}{m c^{2}} \\
& \cdot \int \frac{d r}{\left(1-r_{g} / r\right)\left[\left(E / m c^{2}\right)^{2}-\left(1+M^{2} / m^{2} c^{2} r^{2}\right)\left(1-r_{g} / r\right)\right]^{1 / 2}} \\
\varphi & =\int \frac{M d r}{r^{2} \sqrt{E^{2} / c^{2}-\left(m^{2} c^{2}+M^{2} / r^{2}\right)\left(1-r_{g} / r\right)}}
\end{aligned}
$$

The parameters of the orbits can be obtained by solving the integrals by using some approximate methods. The geodesic equations obtained from the Schwarzschild gravitational metric in the presence of a cosmological constant were solved exactly and expressed in a closed form in [14] as

$$
u=\frac{1}{r}=\frac{4}{\alpha_{S}} \wp(\varphi+\epsilon)+\frac{1}{3 \alpha_{S}},
$$

where $\alpha_{S}=2 G M / c^{2}, \epsilon$ is an arbitrary integration constant, $\wp$ is the Weierstrass function that gives the inversion of the elliptic integral $\int^{U}\left(d U / \sqrt{4 U^{3}-g_{2} U-g_{3}}\right)=\phi$, by the Weierstrass function, $U=\wp(\phi+\epsilon)$. In this approach the exact expression of the perihelion precession is given by $\Delta_{\omega}=2(\omega-$ $\pi)$, where $\omega=\int_{e_{1}}^{\infty}\left(d t / \sqrt{4 t^{3}-g_{2} t-g^{3}}\right)$ and $e_{1}$ is a root of the cubic equation in the integral. The perihelion precession and deflection of light have been investigated in the fourdimensional general spherically symmetric spacetime in [15], where the master equation has also been obtained. As an application of this master equation, the ReissnerNordstorm solution and Clifton-Barrow solution in $f(R)$ gravity have been investigated. The homotopy perturbation method, which was introduced in [16], was applied for calculating the perihelion precession angle of planetary orbits in general relativity in $[17,18]$. The basic ideas behind the homotopy perturbation method are as follows [16]. We start from the nonlinear differential equation $A(u)=g(r), \quad r \in$ $\Omega$, where $A$ is a general differential operator and $g(r)$ is a known analytic function, with the boundary conditions $B(u, \partial u / \partial n)=0 ; r \in \Gamma$, where $B$ is a boundary operator and $\Gamma$ is the boundary of the domain $\Omega$. We assume that the operator $A$ can be divided into two parts $M$ and $N$, and we reformulate our initial equation as $M(u)+N(u)=g(r)$. Then the homotopy $v(r, p): \Omega \times[0,1] \rightarrow \mathbb{R}$ is constructed in the following way: $H(v, p)=(1-p)\left[M(v)-M\left(y_{0}\right)\right]+p[A(v)-$ $g(r)]=0$, where $r \in \Omega$ and $p \in[0,1]$ is an imbedding parameter, and $y_{0}$ is an initial approximation of the equation. Since $0 \leq p \leq 1$, it can be considered as a small parameter, and one can assume that the solution of the equation can be expressed as a power series in $p$ as $v=\sum_{i=0}^{\infty} v_{i} p^{i}$. When $p \rightarrow 1$, then this series becomes the approximate solution of the equation, that is, $u(x)=\lim _{p \rightarrow 1} v=\sum_{i=0}^{\infty} v_{i}$. This series is generally convergent.

The study and the applications of Adomian's Decomposition Method (ADM) [19-22], which allows investigating the solutions of many kinds of ordinary, partial, stochastic differential, and integral equations that describe numerous physical and/or mathematical problems, have attracted a lot of attention in recent years. Historically, the ADM was first proposed and applied in the 1980s [23-25]. An essential advantage of the ADM is that with its use one can obtain analytical approximations to the solutions of a rather wide class of nonlinear (and stochastic) differential and integral equations, without the need of linearization, perturbation, closure approximations, or discretization methods. Usually the application of these methods could lead to the necessity of intensive numerical computation. Moreover, to make solvable and to obtain closed-form analytical solutions of a 
nonlinear problem imply the necessity of introducing some simplifying and restrictive assumptions.

It is important to mention that $\mathrm{ADM}$ can generate the solution of a given equation in the form of a power series. The terms of the series are obtained by recursive relations using the Adomian polynomials. Another important advantage of the ADM is that usually the series solution of the differential equation converges fast, and therefore the use of this method saves a lot of computational time. Moreover, in the ADM there is no need to linearize or discretize the differential equation. Reviews of ADM and its applications in applied mathematics and physics can be found in $[19,20]$, respectively. Many studies have been devoted to the modification and improvement of the ADM in an attempt to increase its accuracy and/or to extend the applicability of the initial method [21, 22, 26-40]. An important improvement of the $\mathrm{ADM}$ is represented by the Laplace-Adomian Decomposition Method [41], in which the Adomian Decomposition Method is applied not to initial equation, but to the Laplace transformed one.

Even that the ADM has been extensively used in the study of many problems in different fields of physics and engineering, it has been applied very little in astronomy, astrophysics, or general relativity, the only exception from this "rule" known to authors being the papers [42, 43]. It is the purpose of the present paper to investigate the equations describing the perihelion precession and light bending in general relativity for static gravitational fields by using the Adomian Decomposition Method, representing a very powerful mathematical method for the investigation of the solutions of nonlinear differential equations. For the geometry outside a compact, stellar type object (the Sun) we adopt some specific static and spherically symmetric vacuum solutions of general relativity. In particular the power series solution of the equation describing the motion of massive and massless particles in Schwarzschild geometry is investigated in detail. As a first step in our analysis we derive the equations of motion for particles in arbitrary spherically symmetric spacetimes, and we develop a general formalism for obtaining the equations of motion that can be used for any given metric. As the next step in our study we adopt the Schwarzschild form of the metric, and we apply the LaplaceAdomian Decomposition Method to obtain its approximate analytical power series solution for both massive and massless particles. We compare our solutions with the exact numerical solutions of the equations of motion, and it turns out that by truncating our series to five terms only we obtain a very good description of the solution of the equation of motion. Moreover, in the first approximation we can reobtain easily the standard expressions of the perihelion precession and the bending angle of light.

The present paper is organized as follows. We derive the equations of motion of massive and massless particles in arbitrary static spherically symmetric spacetimes in Section 2. The application of the Laplace-Adomian Decomposition Method to the case of second-order nonlinear differential equations is presented in Section 3. The power series solution of the equation of motion of massive particles by using the Laplace-Adomian Decomposition Method is obtained in Section 4, where the comparison with the exact numerical solution is also performed. The motion of photons in Schwarzschild geometry is investigated in Section 5. Finally, in Section 6, we discuss and conclude our results.

\section{Particle Motion in Arbitrary Spherically Symmetric Static Spacetimes}

In the following we will restrict our analysis to the case of static and spherically symmetric metrics, given by

$$
d s^{2}=-e^{\nu(r)} d t^{2}+e^{\lambda(r)} d r^{2}+r^{2} d \Omega^{2},
$$

where we have denoted $d \Omega^{2}=d \theta^{2}+\sin ^{2} \theta d \varphi^{2}$ and $\theta$ and $\varphi$ are the standard coordinates on the three-sphere. The time variable takes real values only, $t \in R$ while the radial coordinate $r$ ranges over a finite open interval $r \in\left(r_{\min }, r_{\max }\right)$, so that $-\infty \leq r_{\min } \leq r_{\max } \leq \infty$. Moreover, we also require that the functions $\nu(r)$ and $\lambda(r)$ are strictly positive and that on the interval $\left(r_{\min }, r_{\max }\right)$ they are (at least piecewise) differentiable. This form of the metric is relevant for the study of the dynamics of particles (both massive and massless) in the Solar System.

Important observational evidence for the correctness of the theory of general relativity is provided, at the level of the Solar System, by three fundamental tests, which also allow the testing of its extensions and generalization, as well as of alternative theories of gravitation. These three essential tests are the perihelion precession of Mercury, the deflection of photons by the Sun, and the radar echo delay observations. These three effects have been successfully used to test the Schwarzschild solution of general relativity, as well as other predictions of the theory. However, it is also important to study these physical phenomena in arbitrary static spherically symmetric spacetimes for any given metric. In the present Section, we develop a formalism that can be used for obtaining the equations of motion and compute the perihelion precession and light bending angle in any static spherically symmetric metric. This formalism was first introduced to study the Solar System tests for some modified gravity vacuum solutions in [44-47].

2.1. The Equation of Motion of Massive Test Particles. The geodesic equations of motion of a massive test particle in the gravitational field of the metric given by (5) can be derived with the use of the variational principle

$$
\delta \int \sqrt{e^{v} c^{2} \dot{t}^{2}-e^{\lambda} \dot{r}^{2}-r^{2}\left(\dot{\theta}^{2}+\sin ^{2} \theta \dot{\varphi}^{2}\right)} d s=0,
$$

where by a dot we have denoted $d / d s$. It can be easily checked that the orbit is planar, and therefore without any loss of generality we can take $\theta=\pi / 2$. Hence $\varphi$ is the only the angular coordinate in this problem. Since $t$ and $\varphi$ do not appear explicitly in (6), their conjugate momenta give two constants of motion, denoted by $E$ and $L$, so that

$$
\begin{aligned}
e^{v} c^{2} \dot{t} & =E=\text { constant }, \\
r^{2} \dot{\varphi} & =L=\text { constant. }
\end{aligned}
$$


The constant $E$ gives the energy of the particle, while the constant $L$ is related to its angular momentum.

From the line element (5) we obtain the following equation of motion for $r$ :

$$
\dot{r}^{2}+e^{-\lambda} r^{2} \dot{\varphi}^{2}=e^{-\lambda}\left(e^{\nu} c^{2} \dot{t}^{2}-1\right) .
$$

Substituting $\dot{t}$ and $\dot{\varphi}$ from (7) gives the following relation:

$$
\dot{r}^{2}+e^{-\lambda} \frac{L^{2}}{r^{2}}=e^{-\lambda}\left(\frac{E^{2}}{c^{2}} e^{-v}-1\right) .
$$

We introduce now a new variable $u$, defined as $r=1 / u$, as well as the transformation $d / d s=L u^{2} d / d \varphi$. Then (9) takes the form

$$
\left(\frac{d u}{d \varphi}\right)^{2}+e^{-\lambda} u^{2}=\frac{1}{L^{2}} e^{-\lambda}\left(\frac{E^{2}}{c^{2}} e^{-\nu}-1\right) .
$$

We represent formally $e^{-\lambda}$ as $e^{-\lambda}=1-f(u)$, thus obtaining

$$
\begin{aligned}
\left(\frac{d u}{d \varphi}\right)^{2}+u^{2} & =f(u) u^{2}+\frac{E^{2}}{c^{2} L^{2}} e^{-\nu-\lambda}-\frac{1}{L^{2}} e^{-\lambda} \\
& \equiv G(u) .
\end{aligned}
$$

We take the derivative of the above equation with respect to $\varphi$, which gives

$$
\frac{d^{2} u}{d \varphi^{2}}+u=F(u)
$$

where

$$
F(u)=\frac{1}{2} \frac{d G(u)}{d u}
$$

Equation (12) gives the equation of motion of a particle in an arbitrary spherically symmetric geometry.

2.1.1. The Precession of the Perihelion. The root of the equation $u_{0}=F\left(u_{0}\right)$ gives a circular orbit with $u=u_{0}$. Any deviation $\delta=u-u_{0}$ from it can be obtained from the substitution of $u=u_{0}+\delta$ into (12), which gives the equation

$$
\frac{d^{2} \delta}{d \varphi^{2}}+u_{0}+\delta=F\left(u_{0}+\delta\right) .
$$

In the first order of approximation $F\left(u_{0}+\delta\right) \approx F\left(u_{0}\right)+$ $\left.(d F / d u)\right|_{u=u_{0}} \delta+O\left(\delta^{2}\right)$ and hence

$$
\frac{d^{2} \delta}{d \varphi^{2}}+\left[1-\left(\frac{d F}{d u}\right)_{u=u_{0}}\right] \delta=O\left(\delta^{2}\right) .
$$

Therefore, to first order in $\delta$, the trajectory of the massive particle can be obtained as

$$
\delta=\delta_{0} \cos \left(\sqrt{1-\left(\frac{d F}{d u}\right)_{u=u_{0}}} \varphi+\beta\right),
$$

where $\delta_{0}$ and $\beta$ are two arbitrary constants of integration. The angles for which $r$ is minimum are the angles of the perihelia of the orbit. Therefore they are determined from the condition that $u$ or $\delta$ is maximum. Hence, from one perihelion to the next the orbital angle varies by a quantity $\Delta \varphi$, given by

$$
\Delta \varphi=\frac{2 \pi}{\sqrt{1-(d F / d u)_{u=u_{0}}}}=\frac{2 \pi}{1-\sigma} .
$$

The parameter $\sigma$ introduced in the previous equation is called the perihelion advance. From a physical point of view it represents the rate of advance of the perihelion after one rotation. As the test particle advances through $\varphi$ radians in its orbit, its perihelion precesses by $\sigma \Delta \varphi$ radians. From (17), $\sigma$ can be expressed as

$$
\sigma=1-\sqrt{1-\left(\frac{d F}{d u}\right)_{u=u_{0}}}
$$

or for small $(d F / d u)_{u=u_{0}}$, as

$$
\sigma=\frac{1}{2}\left(\frac{d F}{d u}\right)_{u=u_{0}}
$$

For a complete rotation of the planet we obtain $\varphi \approx 2 \pi(1+$ $\sigma)$. Hence the advance of the perihelion is

$$
\delta \varphi=\varphi-2 \pi \approx 2 \pi \sigma .
$$

To be able to obtain effective estimations of the perihelion precession we must know the expression of the angular momentum of the particle $L$ as a function of the geometric parameters of the orbit. We will obtain now the expression of $L$ in the Newtonian limit [44-47].

Let us assume that the planet moves on a Keplerian ellipse, with semiaxes $\bar{a}$ and $\bar{b}$, respectively, where $\bar{b}=\bar{a} \sqrt{1-e^{2}}$ and by $e$ we have denoted the eccentricity of the orbit. The ellipse has a surface area $\pi \bar{a} \bar{b}$. The oriented surface area of the ellipse is $\overrightarrow{d A}=(\vec{r} \times \overrightarrow{d r}) / 2$, and consequently the areolar velocity of the planet is given by $|\overrightarrow{d A} / d t|=|\vec{r} \times d \vec{r}| / 2=r^{2}(d \varphi / d t) / 2 \approx$ $\pi \bar{a}^{2} \sqrt{1-e^{2}} / T$, where $T$ is the period of the planetary motion. On the other hand $T$ can be obtained from Kepler's third law as $T^{2}=4 \pi^{2} \bar{a}^{3} / G M$ [48]. In the Newtonian limit of small velocities $d s \approx c d t$, and the conservation equation of the angular momentum reduces to $r^{2} d \varphi / d t=c L$. Hence we obtain first $L=2 \pi \bar{a}^{2} \sqrt{1-e^{2}} / c T$, and hence

$$
\frac{1}{L^{2}}=\frac{c^{2}}{G M \bar{a}\left(1-e^{2}\right)} \text {. }
$$

2.1.2. The Equation of Motion of Massive Particles in Schwarzschild Geometry. As a first astronomical application of the formalism introduced in the previous section we obtain the precession of the perihelion of a planet in the Schwarzschild geometry, with

$$
e^{\nu}=e^{-\lambda}=1-\frac{2 G M}{c^{2} r}=1-\left(\frac{2 G M}{c^{2}}\right) u .
$$


Then we immediately obtain $f(u)=\left(2 G M / c^{2}\right) u$. On the other hand since for this geometry $\nu+\lambda=0$, we easily find

$$
G(u)=\frac{2 G M}{c^{2}} u^{3}+\frac{1}{L^{2}}\left(\frac{E^{2}}{c^{2}}-1\right)+\frac{2 G M}{c^{2} L^{2}} u
$$

and

$$
F(u)=3 \frac{G M}{c^{2}} u^{2}+\frac{G M}{c^{2} L^{2}},
$$

respectively. Therefore the equation of motion of a massive test particle in Schwarzschild geometry is given by

$$
\frac{d^{2} u}{d \varphi^{2}}+u=3 \frac{G M}{c^{2}} u^{2}+\frac{G M}{c^{2} L^{2}}
$$

The radius $u=u_{0}$ of the circular orbit is found as the solution of the algebraic equation

$$
u_{0}=3 \frac{G M}{c^{2}} u_{0}^{2}+\frac{G M}{c^{2} L^{2}}
$$

with the only physically acceptable solution given by

$$
u_{0}=\frac{1 \pm \sqrt{1-12 G^{2} M^{2} / c^{4} L^{2}}}{6 G M / c^{2}} \approx \frac{G M}{c^{2} L^{2}} .
$$

Therefore

$$
\delta \varphi=\pi\left(\frac{d F}{d u}\right)_{u=u_{0}}=\frac{6 \pi G M}{c^{2} \bar{a}\left(1-e^{2}\right)},
$$

which is the standard general relativistic result [13].

2.2. Equation of Motions of Photons and the Deflection of Light. In a gravitational field a photon follows a null geodesic, given by $d s^{2}=0$. In this case the affine parameter along the trajectory of the photon can be taken as an arbitrary quantity $\tilde{\lambda}$. In the following we denote again by a dot the derivatives with respect to it. Similar to the case of the motion of massive particles we have two constants of motion, the energy $E$ and the angular momentum $L$, which can be obtained from (7).

The equation of motion of the photon is given by

$$
\dot{r}^{2}+e^{-\lambda} r^{2} \dot{\varphi}^{2}=e^{\nu-\lambda} c^{2} \dot{t}^{2}
$$

By using the constants of motion the above equation can be transformed into

$$
\dot{r}^{2}+e^{-\lambda} \frac{L^{2}}{r^{2}}=\frac{E^{2}}{c^{2}} e^{-\nu-\lambda} .
$$

We change now the independent variable $r$ to $u=1 / r$. With the use of the conservation equations we eliminate the derivative with respect to the affine parameter, thus obtaining

$$
\left(\frac{d u}{d \varphi}\right)^{2}+u^{2}=f(u) u^{2}+\frac{1}{c^{2}} \frac{E^{2}}{L^{2}} e^{-v-\lambda} \equiv P(u) .
$$

We take the derivative of (31) with respect to $\varphi$, and thus we find the basic equation of the photon in an arbitrary static spherically symmetric geometry, as given by

$$
\frac{d^{2} u}{d \varphi^{2}}+u=V(u)
$$

where we have denoted

$$
V(u)=\frac{1}{2} \frac{d P(u)}{d u} .
$$

In the particular case of the Schwarzschild geometry we have $v+\lambda=0$ and $f(u)=\left(2 G M / c^{2}\right) u$, giving $P(u)=$ $\left(2 G M / c^{2}\right) u^{3}$ and $V(u)=\left(3 G M / c^{2}\right) u^{2}$, respectively. Hence the equation of motion of photons in the Schwarzschild metric is obtained as

$$
\frac{d^{2} u}{d \varphi^{2}}+u=\frac{3 G M}{c^{2}} u^{2}
$$

2.2.1. The Deflection Angle of Light. In the lowest order of approximation we can neglect the term on the right-hand side of (32). Then the solution is given by a straight line,

$$
u=\frac{\cos \varphi}{R},
$$

whereby $R$ we have denoted the distance of the closest approach to the central massive gravitating object. In the next order of approximation (35) is substituted into the right-hand side of (32). Hence the equation of the trajectory is given by a second-order linear inhomogeneous differential equation,

$$
\frac{d^{2} u}{d \varphi^{2}}+u=V\left(\frac{\cos \varphi}{R}\right)
$$

which has a general solution given by $u=u(\varphi)$. The photons travel towards the star from infinity at the asymptotic angle $\varphi=-(\pi / 2+\varepsilon)$ and are deflected to infinity at the asymptotic angle $\varphi=\pi / 2+\varepsilon$. The angle $\varepsilon$ can be computed by solving the algebraic equation $u(\pi / 2+\varepsilon)=0$. For the total deflection angle of the photon beam we find $\delta=2 \varepsilon$.

(a) The Deflection of Light in Schwarzschild Geometry. We consider now the case of the Schwarzschild geometry. In the lowest order of approximation from (35) and (36) we obtain for the photon trajectory the second-order linear differential equation

$$
\frac{d^{2} u}{d \varphi^{2}}+u=\frac{3 G M}{c^{2} R^{2}} \cos ^{2} \varphi=\frac{3 G M}{2 c^{2} R^{2}}(1+\cos 2 \varphi),
$$

having the general solution given by

$$
u=\frac{\cos \varphi}{R}+\frac{3 G M}{2 c^{2} R^{2}}\left(1-\frac{1}{3} \cos 2 \varphi\right) .
$$

By substituting $\varphi=\pi / 2+\varepsilon, u=0$ into (38) we easily find

$$
\varepsilon=\frac{2 G M}{c^{2} R},
$$


where we have used the simple trigonometric relations $\cos (\pi / 2+\varepsilon)=-\sin \varepsilon, \cos (\pi+2 \varepsilon)=-\cos 2 \varepsilon$, and the approximations $\sin \varepsilon \approx \varepsilon$ and $\cos 2 \varepsilon \approx 1$, respectively. The total deflection angle of light in the Schwarzschild geometry is thus $\delta=2 \varepsilon=4 G M / c^{2} R$, a well-known result in general relativity [13].

\section{The Laplace-Adomian Method for Nonlinear Second-Order Ordinary Differential Equations}

3.1. The General Formalism. Let us consider a nonlinear differential equation of the form

$$
y^{\prime \prime}+\omega^{2} y+b^{2}+g(y)=0,
$$

where $\omega$ and $b$ are constants and $g$ is an arbitrary nonlinear function of dependent variable $y$. Equation (40) must be integrated with the initial conditions $y(0)=y_{0}=a$ and $y^{\prime}(0)=0$, respectively.

In the Laplace-Adomian method we first apply the Laplace transformation operator $\mathscr{L}$ to (40), thus obtaining

$$
\mathscr{L}\left[y^{\prime \prime}\right]+\omega^{2} \mathscr{L}[y]+\mathscr{L}\left[b^{2}\right]+\mathscr{L}[g(y)]=0 .
$$

With the use of the properties of the Laplace transformation we easily find

$$
\begin{aligned}
& \left(s^{2}+\omega^{2}\right) \mathscr{L}[y]-s y(0)-y^{\prime}(0)+\frac{b^{2}}{s}+\mathscr{L}[g(y)] \\
& \quad=0 .
\end{aligned}
$$
obtain

With the use of the initial conditions for our problem we

$$
\mathscr{L}[y]=\frac{a s}{s^{2}+\omega^{2}}-\frac{b^{2}}{s\left(s^{2}+\omega^{2}\right)}-\frac{1}{s^{2}+\omega^{2}} \mathscr{L}[g(y)] .
$$

As a next step in our analysis we assume that the solution can be represented in the form of an infinite series,

$$
y(x)=\sum_{n=0}^{\infty} y_{n}(x)
$$

where the terms $y_{n}(x)$ are computed recursively. As for the nonlinear operator $g(y)$, it is decomposed as

$$
g(y)=\sum_{n=0}^{\infty} A_{n}
$$

where $A_{n}$ 's are the so-called Adomian polynomials, defined generally as [20]

$$
A_{n}=\left.\frac{1}{n !} \frac{d^{n}}{d \epsilon^{n}} f\left(\sum_{i=0}^{\infty} \epsilon^{i} y_{i}\right)\right|_{\epsilon=0} .
$$

The first five Adomian polynomials can be obtained in the following form:

$$
A_{0}=f\left(y_{0}\right),
$$

$$
\begin{aligned}
A_{1}= & y_{1} f^{\prime}\left(y_{0}\right), \\
A_{2}= & y_{2} f^{\prime}\left(y_{0}\right)+\frac{1}{2} y_{1}^{2} f^{\prime \prime}\left(y_{0}\right), \\
A_{3}= & y_{3} f^{\prime}\left(y_{0}\right)+y_{1} y_{2} f^{\prime \prime}\left(y_{0}\right)+\frac{1}{6} y_{1}^{3} f^{\prime \prime \prime}\left(y_{0}\right), \\
A_{4}= & y_{4} f^{\prime}\left(y_{0}\right)+\left[\frac{1}{2 !} y_{2}^{2}+y_{1} y_{3}\right] f^{\prime \prime}\left(y_{0}\right) \\
& +\frac{1}{2 !} y_{1}^{2} y_{2} f^{\prime \prime \prime}\left(y_{0}\right)+\frac{1}{4 !} y_{1}^{4} f^{(\mathrm{iv})}\left(y_{0}\right) .
\end{aligned}
$$

Substituting (44) and (45) into (43) we obtain

$$
\begin{aligned}
\mathscr{L}\left[\sum_{n=0}^{\infty} y_{n}(x)\right]= & \frac{a s}{s^{2}+\omega^{2}}-\frac{b^{2}}{s\left(s^{2}+\omega^{2}\right)} \\
& -\frac{1}{s^{2}+\omega^{2}} \mathscr{L}\left[\sum_{n=0}^{\infty} A_{n}\right] .
\end{aligned}
$$

Matching both sides of (52) yields the following iterative algorithm for the power series solution of (40),

$$
\begin{gathered}
\mathscr{L}\left[y_{0}\right]=\frac{a s}{s^{2}+\omega^{2}}-\frac{b^{2}}{s\left(s^{2}+\omega^{2}\right)}, \\
\mathscr{L}\left[y_{1}\right]=-\frac{1}{s^{2}+\omega^{2}} \mathscr{L}\left[A_{0}\right], \\
\mathscr{L}\left[y_{2}\right]=-\frac{1}{s^{2}+\omega^{2}} \mathscr{L}\left[A_{1}\right], \\
\ldots \\
\mathscr{L}\left[y_{k+1}\right]=-\frac{1}{s^{2}+\omega^{2}} \mathscr{L}\left[A_{k}\right] .
\end{gathered}
$$

By applying the inverse Laplace transformation to (53), we obtain the value of $y_{0}$. Substituting $y_{0}$ into (47) to find the first Adomian polynomial $A_{0}$, then we substitute $A_{0}$ into (54), and we evaluate the Laplace transform of the quantities on the right-hand side of it. The application of the inverse Laplace transformation yields then the value of $y_{1}$. The other terms $y_{2}, y_{3}, \ldots, y_{k+1}$ can be computed recursively in a similar step by step approach.

3.2. The Particular Case $g(y)=\sum_{l=0}^{m} a_{l+2} y^{l+2}$. Let us consider a nonlinear differential equation of the form

$$
\frac{d^{2} y}{d x^{2}}+\omega^{2} y+b^{2}+\sum_{l=0}^{m} a_{l+2} y^{l+2}=0
$$

where $\omega, b$, and $a_{l+2}, l=0, \ldots, m$, are constants. Equation (58) must be integrated with the initial conditions $y(0)=$ $y_{0}=a$ and $y^{\prime}(0)=0$, respectively. By applying the LaplaceAdomian method we first take the Laplace transform to (58), thus obtaining

$$
\begin{aligned}
& \mathscr{L}\left(\frac{d^{2} y}{d x^{2}}\right)+\omega^{2} \mathscr{L}(y)+b^{2} \mathscr{L}(1)+\sum_{l=0}^{m} a_{l+2} \mathscr{L}\left[y^{l+2}\right] \\
& \quad=0 .
\end{aligned}
$$


Now we easily find

$$
\begin{aligned}
\mathscr{L}(y)\left(s^{2}+\omega^{2}\right)= & s y(0)+y^{\prime}(0)-\frac{b^{2}}{s} \\
& -\sum_{l=0}^{m} a_{l+2} \mathscr{L}\left[y^{l+2}\right]=0,
\end{aligned}
$$

thus obtaining

$$
\begin{aligned}
\mathscr{L}(y)= & \frac{s y(0)+y^{\prime}(0)}{s^{2}+\omega^{2}}-\frac{b^{2}}{s\left(s^{2}+\omega^{2}\right)} \\
& -\frac{1}{s^{2}+\omega^{2}} \sum_{l=0}^{m} a_{l+2} \mathscr{L}\left[y^{l+2}\right] .
\end{aligned}
$$

From (61) we obtain $y(x)$ in the form

$$
\begin{aligned}
y(x)= & \mathscr{L}^{-1}\left[\frac{s y(0)+y^{\prime}(0)}{s^{2}+\omega^{2}}-\frac{b^{2}}{s\left(s^{2}+\omega^{2}\right)}\right] \\
& -\mathscr{L}^{-1}\left[\frac{1}{s^{2}+\omega^{2}} \sum_{l=0}^{m} a_{l+2} \mathscr{L}\left[y^{l+2}\right]\right] .
\end{aligned}
$$

We assume a power series solution for $y(x)$ as $y(x)=$ $\sum_{n=0}^{\infty} y_{n}(x)$, and we write the nonlinear terms as

$$
y^{l+2}=\sum_{n=0}^{\infty} A_{n, l+2}(x)
$$

where $A_{n, l+2}$ are the Adomian polynomial corresponding to $y^{l+2}$. Then we obtain

$$
\begin{aligned}
\sum_{n=0}^{\infty} y_{n}(x) & \\
= & \mathscr{L}^{-1}\left[\frac{s y(0)+y^{\prime}(0)}{s^{2}+\omega^{2}}-\frac{b^{2}}{s\left(s^{2}+\omega^{2}\right)}\right] \\
& -\mathscr{L}^{-1}\left\{\frac{1}{s^{2}+\omega^{2}} \sum_{l=0}^{m} a_{l+2} \mathscr{L}\left[\sum_{n=0}^{\infty} A_{n, l+2}(x)\right]\right\} .
\end{aligned}
$$

We rewrite (64) in the form

$$
\begin{aligned}
y_{0}(x) & +\sum_{n=0}^{\infty} y_{n+1}(x) \\
= & \mathscr{L}^{-1}\left[\frac{s y(0)+y^{\prime}(0)}{s^{2}+\omega^{2}}-\frac{b^{2}}{s\left(s^{2}+\omega^{2}\right)}\right] \\
& -\sum_{n=0}^{\infty} \mathscr{L}^{-1}\left\{\frac{1}{s^{2}+\omega^{2}} \sum_{l=0}^{m} a_{l+2} \mathscr{L}\left[A_{n, l+2}(x)\right]\right\} .
\end{aligned}
$$

Equation (65) can be written as the recursive relations

$$
y_{0}(x)=\mathscr{L}^{-1}\left[\frac{s y(0)+y^{\prime}(0)}{s^{2}+\omega^{2}}-\frac{b^{2}}{s\left(s^{2}+\omega^{2}\right)}\right]
$$

$$
y_{k+1}(x)=-\mathscr{L}^{-1}\left\{\frac{1}{s^{2}+\omega^{2}} \sum_{l=0}^{m} a_{l+2} \mathscr{L}\left[A_{k, l+2}(x)\right]\right\} .
$$

For the function $y^{l+2}$ a few Adomian polynomials are

$$
\begin{aligned}
A_{0, l+2}= & y_{0}^{l+2}, \\
A_{1, l+2}= & (l+2) y_{1} y_{0}^{l+1}, \\
A_{2, l+2}= & (l+2) y_{2} y_{0}^{l+1}+(l+1)(l+2) \frac{y_{1}^{2}}{2 !} y_{0}^{l}, \\
A_{3, l+2}= & (l+2) y_{3} y_{0}^{l+1}+(l+1)(l+2) y_{1} y_{2} y_{0}^{l} \\
& +l(l+1)(l+2) \frac{y_{1}^{3}}{3 !} y_{0}^{l-1} .
\end{aligned}
$$

For $k=0$ we obtain the first-order approximation to the solution as

$$
\begin{aligned}
& y_{1}(x) \\
& =-\mathscr{L}^{-1}\left\{\frac{\mathscr{L}\left[a_{2} A_{0,2}(x)+a_{3} A_{0,3}(x)+a_{4} A_{0,4}(x)+\cdots\right]}{s^{2}+\omega^{2}}\right\} \\
& =-\mathscr{L}^{-1}\left\{\frac{\mathscr{L}\left(a_{2} y_{0}^{2}+a_{3} y_{0}^{3}+a_{4} y_{0}^{4}+\cdots\right)}{s^{2}+\omega^{2}}\right\} .
\end{aligned}
$$

For $k=1$ we find

$$
\begin{aligned}
& y_{2}(x) \\
& =-\mathscr{L}^{-1}\left\{\frac{\mathscr{L}\left[a_{2} A_{1,2}(x)+a_{3} A_{1,3}(x)+a_{4} A_{1,4}(x)+\cdots\right]}{s^{2}+\omega^{2}}\right\} \\
& =-\mathscr{L}^{-1}\left\{\frac{\mathscr{L}\left(2 a_{2} y_{1} y_{0}+3 a_{3} y_{1} y_{0}^{2}+4 a_{4} y_{1} y_{0}^{3}+\cdots\right)}{s^{2}+\omega^{2}}\right\} . \\
& k=2 \text { gives }
\end{aligned}
$$

$$
y_{3}(x)=-\mathscr{L}^{-1}\left\{\frac{\mathscr{L}\left[a_{2} A_{2,2}(x)+a_{3} A_{2,3}(x)+a_{4} A_{2,4}(x)+\cdots\right]}{s^{2}+\omega^{2}}\right\}
$$




$$
=-\mathscr{L}^{-1}\left\{\frac{\mathscr{L}\left[a_{2}\left(2 y_{2} y_{0}+y_{1}^{2}\right)+3 a_{3}\left(y_{2} y_{0}^{2}+y_{1}^{2} y_{0}\right)+a_{4}\left(4 y_{2} y_{0}^{3}+6 y_{1}^{2} y_{0}^{2}\right)+\cdots\right]}{s^{2}+\omega^{2}}\right\} .
$$

Finally, for $k=3$ we obtain

$$
\begin{aligned}
y_{4}(x) & \\
& =-\mathscr{L}^{-1}\left\{\frac{\mathscr{L}\left[a_{2} A_{3,2}(x)+a_{3} A_{3,3}(x)+a_{4} A_{3,4}(x)+\cdots\right]}{s^{2}+\omega^{2}}\right\} \\
& =-\mathscr{L}^{-1}\left\{\frac { 1 } { s ^ { 2 } + \omega ^ { 2 } } \mathscr { L } \left[2 a_{2}\left(y_{3} y_{0}+y_{1} y_{2}\right)\right.\right. \\
& +a_{3}\left(3 y_{3} y_{0}^{2}+6 y_{1} y_{2} y_{0}+y_{1}^{3}\right) \\
& \left.\left.+a_{4}\left(4 y_{3} y_{0}^{3}+12 y_{1} y_{2} y_{0}^{2}+4 y_{1}^{3} y_{0}\right)+\cdots\right]\right\} .
\end{aligned}
$$

Hence we have obtained the truncated power series solution of (58) as given by

$$
\begin{aligned}
y(x)= & \sum_{n=0}^{\infty} y_{n}(x) \\
= & y_{0}(x)+y_{1}(x)+y_{2}(x)+y_{3}(x)+y_{4}(x) \\
& +\cdots .
\end{aligned}
$$

\section{The Solution of the Equation of the Motion of the Massive Test Particles in Schwarzschild Geometry by the Laplace-Adomian Decomposition Method}

In the following we will use a system of units with $G=c=1$. Then (25), describing the motion of a massive test particle in the Schwarzschild geometry, takes the form

$$
\frac{d^{2} u}{d \varphi^{2}}+u=\frac{M}{L^{2}}+3 M u^{2} .
$$

In order to simplify the mathematical formalism we rescale the function $u$ so that

$$
u=\frac{1}{3 M} U
$$

Thus (78) becomes

$$
\frac{d^{2} U}{d \varphi^{2}}+U=b^{2}+U^{2}
$$

where we have denoted $b^{2}=3 M^{2} / L^{2}$. Equation (80) must be solved with the initial conditions $U(0)=3 M u(0)=a$ and $U^{\prime}(0)=0$, respectively.

4.1. Power Series Solution of the Equation of Motion. Assume that the solution of (80) can be obtained in power series form,

$$
U(\varphi)=\sum_{n=0}^{\infty} U_{n}(\varphi)
$$

Now taking Laplace transform to (80) yields

$$
\mathscr{L}\left[\frac{d^{2} U}{d \varphi^{2}}\right]+\mathscr{L}[U]=b^{2} \mathscr{L}[1]+\mathscr{L}\left[U^{2}\right] .
$$

Hence we obtain

$$
\begin{aligned}
& s^{2} \mathscr{L}(U)-s U(0)-U^{\prime}(0)+\mathscr{L}(U)=\frac{b^{2}}{s}+\mathscr{L}\left[U^{2}\right], \\
& \mathscr{L}(U) \\
& \quad=\frac{s U(0)+U^{\prime}(0)}{s^{2}+1}+\frac{b^{2}}{s\left(s^{2}+1\right)}+\frac{1}{s^{2}+1} \mathscr{L}\left[U^{2}\right] .
\end{aligned}
$$

We write down a few Adomian polynomials for $U^{2}$,

$$
\begin{aligned}
& A_{0}=U_{0}^{2}, \\
& A_{1}=2 U_{1} U_{0}, \\
& A_{2}=2 U_{2} U_{0}+U_{1}^{2} . \\
& A_{3}=2 U_{3} U_{0}+2 U_{1} U_{2},
\end{aligned}
$$

Substituting (81) and $U^{2}=\sum_{n=0}^{\infty} A_{n}(\varphi)$ into (84) gives the relation

$$
\begin{aligned}
\mathscr{L}\left[\sum_{n=0}^{\infty} U_{n}(\varphi)\right]= & \frac{s U(0)+U^{\prime}(0)}{s^{2}+1}+\frac{b^{2}}{s\left(s^{2}+1\right)} \\
& +\frac{1}{s^{2}+1} \mathscr{L}\left[\sum_{n=0}^{\infty} A_{n}(\varphi)\right],
\end{aligned}
$$

or equivalently,

$$
\begin{aligned}
U_{0}(\varphi) & +\sum_{n=1}^{\infty} U_{n}(\varphi)=U_{0}(\varphi)+\sum_{n=0}^{\infty} U_{n+1}(\varphi) \\
= & \mathscr{L}^{-1}\left[\frac{s U(0)+U^{\prime}(0)}{s^{2}+1}+\frac{b^{2}}{s\left(s^{2}+1\right)}\right] \\
& +\sum_{n=0}^{\infty} \mathscr{L}^{-1}\left[\frac{\mathscr{L}\left[A_{n}(\varphi)\right]}{s^{2}+1}\right] .
\end{aligned}
$$

Next we rewrite (90) in the recursive forms

$$
\begin{gathered}
U_{0}(\varphi)=\mathscr{L}^{-1}\left[\frac{s U(0)+U^{\prime}(0)}{s^{2}+1}+\frac{b^{2}}{s\left(s^{2}+1\right)}\right], \\
\ldots, \\
U_{k+1}(\varphi)=\mathscr{L}^{-1}\left[\frac{\mathscr{L}\left[A_{k}(\varphi)\right]}{s^{2}+1}\right] .
\end{gathered}
$$


With the help of the explicit expressions of the Adomian polynomials, we obtain

$$
\begin{aligned}
& U_{0}(\varphi)=\left(a-b^{2}\right) \cos \varphi+b^{2}, \\
& A_{0}=U_{0}^{2}=\left[\left(a-b^{2}\right) \cos \varphi+b^{2}\right]^{2}, \\
& U_{1}(\varphi)=\mathscr{L}^{-1}\left[\frac{\mathscr{L}\left[A_{0}(\varphi)\right]}{s^{2}+1}\right]=\mathscr{L}^{-1}\left[\frac{\mathscr{L}\left[U_{0}^{2}\right]}{s^{2}+1}\right], \\
& k=0,
\end{aligned}
$$$$
U_{1}(\varphi)=\frac{1}{6}\left\{-2\left(a^{2}-2 a b^{2}+4 b^{4}\right) \cos \varphi+3\left(a^{2}\right.\right.
$$$$
\left.-2 a b^{2}+3 b^{4}\right)+\left(a-b^{2}\right)\left[\left(b^{2}-a\right) \cos (2 \varphi)\right.
$$$$
\left.\left.+6 b^{2} \varphi \sin \varphi\right]\right\} \text {, }
$$$$
\begin{aligned}
A_{1} & =2 U_{1} U_{0}=\frac{1}{3}\left[\left(a-b^{2}\right) \cos \varphi+b^{2}\right]\left\{-2\left(a^{2}\right.\right. \\
& \left.-2 a b^{2}+4 b^{4}\right) \cos \varphi+3\left(a^{2}-2 a b^{2}+3 b^{4}\right)+(a \\
& \left.\left.-b^{2}\right)\left[\left(b^{2}-a\right) \cos (2 \varphi)+6 b^{2} \varphi \sin \varphi\right]\right\},
\end{aligned}
$$$$
U_{2}(\varphi)=\mathscr{L}^{-1}\left[\frac{\mathscr{L}\left[A_{1}(\varphi)\right]}{s^{2}+1}\right]=2 \mathscr{L}^{-1}\left[\frac{\mathscr{L}\left[U_{1} U_{0}\right]}{s^{2}+1}\right],
$$$$
k=1 \text {, }
$$

$$
\begin{aligned}
& U_{2}(\varphi)=\frac{1}{144}\left\{16\left(a^{2}-5 a b^{2}+7 b^{4}\right)\left(a-b^{2}\right)\right. \\
& \cdot \cos (2 \varphi)+\cos \varphi\left[29 a^{3}-183 a^{2} b^{2}\right. \\
& \left.+3 a b^{4}\left(125-24 \varphi^{2}\right)+b^{6}\left(72 \varphi^{2}-509\right)\right] \\
& +12 \varphi\left(5 a^{3}-19 a^{2} b^{2}+41 a b^{4}-39 b^{6}\right) \times \sin \varphi \\
& -48\left(a^{3}-6 a^{2} b^{2}+12 a b^{4}-13 b^{6}\right)-48 \varphi\left(b^{3}\right. \\
& \left.-a b)^{2} \sin (2 \varphi)+3\left(a-b^{2}\right)^{3} \cos (3 \varphi)\right\},
\end{aligned}
$$

$$
\begin{aligned}
U_{3}(\varphi) & =\mathscr{L}^{-1}\left[\frac{\mathscr{L}\left[A_{2}(\varphi)\right]}{s^{2}+1}\right] \\
& =\mathscr{L}^{-1}\left[\frac{\mathscr{L}\left[2 U_{2} U_{0}+U_{1}^{2}\right]}{s^{2}+1}\right], \quad k=2,
\end{aligned}
$$

$$
\begin{aligned}
& U_{3}(\varphi)=\frac{1}{432}\left\{-12 \varphi\left(5 a^{3}-23 a^{2} b^{2}+73 a b^{4}-79 b^{6}\right)\right. \\
& \cdot\left(a-b^{2}\right) \sin (2 \varphi)-3 \varphi \sin \varphi\left[60 a^{4}-449 a^{3} b^{2}\right. \\
& +1335 a^{2} b^{4}+3 a b^{6}\left(8 \varphi^{2}-721\right) \\
& \left.+b^{8}\left(1721-24 \varphi^{2}\right)\right]+\cos \varphi \times\left[-119 a^{4}\right. \\
& +4 a^{3} b^{2}\left(206-45 \varphi^{2}\right)+3 a^{2} b^{4}\left(204 \varphi^{2}-961\right)
\end{aligned}
$$

$$
\begin{aligned}
& U_{4}(\varphi)=\mathscr{L}^{-1}\left[\frac{\mathscr{L}\left[A_{3}(\varphi)\right]}{s^{2}+1}\right] \\
&=2 \mathscr{L}^{-1}\left[\frac{\mathscr{L}\left[U_{3} U_{0}+U_{1} U_{2}\right]}{s^{2}+1}\right], \quad k=3,
\end{aligned}
$$$$
U_{4}(\varphi)=\frac{1}{20736}\left\{32\left(2 a^{2}-13 a b^{2}+17 b^{4}\right)\left(a-b^{2}\right)^{3}\right.
$$$$
\cdot \cos (4 \varphi)+108 \varphi\left(5 a^{3}-27 a^{2} b^{2}+105 a b^{4}\right.
$$$$
\left.-119 b^{6}\right)\left(a-b^{2}\right)^{2} \sin (3 \varphi)+36\left(a-b^{2}\right) \cos (3 \varphi)
$$$$
\cdot\left(31 a^{4}-196 a^{3} b^{2}+642 a^{2} b^{4}-1036 a b^{6}\right.
$$$$
\left.-54 b^{4} \varphi^{2}\left(a-b^{2}\right)^{2}+595 b^{8}\right)+24 \varphi \sin \varphi\left[580 a^{5}\right.
$$$$
-4938 a^{4} b^{2}+a^{3} b^{4}\left(20129-180 \varphi^{2}\right)
$$$$
+7 a^{2} b^{6}\left(84 \varphi^{2}-6583\right)+3 a b^{8}\left(20397-428 \varphi^{2}\right)
$$$$
\left.+5 b^{10}\left(204 \varphi^{2}-8221\right)\right]+192 \varphi \sin (2 \varphi)\left[20 a^{5}\right.
$$$$
-223 a^{4} b^{2}+884 a^{3} b^{4}-2036 a^{2} b^{6}+2608 a b^{8}
$$$$
\left.+24 b^{6} \varphi^{2}\left(a-b^{2}\right)^{2}-1289 b^{10}\right]+384 \cos (2 \varphi)
$$$$
\cdot\left[16 a^{5}+a^{4} b^{2}\left(15 \varphi^{2}-152\right)+8 a^{3} b^{4}\left(74-9 \varphi^{2}\right)\right.
$$$$
+8 a^{2} b^{6}\left(27 \varphi^{2}-164\right)+104 a b^{8}\left(16-3 \varphi^{2}\right)
$$$$
\left.+b^{10}\left(153 \varphi^{2}-862\right)\right]-3 \cos (\varphi)
$$$$
\cdot\left[a^{5}\left(600 \varphi^{2}-2357\right)+a^{4} b^{2}\left(23209-5880 \varphi^{2}\right)\right.
$$$$
+2 a^{3} b^{4}\left(15588 \varphi^{2}-49577\right)
$$$$
+2 a^{2} b^{6}\left(126457-39324 \varphi^{2}\right)
$$$$
+a b^{8}\left(96 \varphi^{2}\left(1235-3 \varphi^{2}\right)-362713\right)
$$$$
\left.+b^{10}\left(288 \varphi^{4}-83088 \varphi^{2}+298693\right)\right]-288\left(50 a^{5}\right.
$$$$
-475 a^{4} b^{2}+1934 a^{3} b^{4}-4604 a^{2} b^{6}+6208 a b^{8}
$$ 


$$
\begin{aligned}
& \left.-4337 b^{10}\right)-192 b^{2} \varphi\left(a-b^{2}\right)^{4} \sin (4 \varphi)+5(a \\
& \left.\left.-b^{2}\right)^{5} \cos (5 \varphi)\right\} .
\end{aligned}
$$

The power series solution of the equation of motion of the massive test particles in Schwarzschild geometry is thus given by

$$
\begin{aligned}
U(\varphi)= & U_{0}(\varphi)+U_{1}(\varphi)+U_{2}(\varphi)+U_{3}(\varphi)+U_{4}(\varphi) \\
& +\cdots .
\end{aligned}
$$

4.2. Comparison with the Exact Numerical Solution. In order to estimate the results obtained by the Laplace-Adomian Decomposition Method, in Figure 1 we present the comparison between the exact numerical solution of (80) and the analytical, power series representation, given by (105), for $b=0.15, b^{2}=0.0225$, and $a=0.001$, respectively.

The absolute difference $\Delta(\varphi)$, defined as

$$
\Delta(\varphi)=\left.U(\varphi)\right|_{\text {num }}-\left.U(\varphi)\right|_{\text {LADM }}
$$

between the numerical solution $\left.U(\varphi)\right|_{\text {num }}$ and the truncated Laplace-Adomian power series solution, is represented in Figure 2.
4.3. Application: The Motion of Planet Mercury. In order to integrate the equation of motion of the massive test particles in Schwarzschild geometry we need to know the initial conditions of the motion. For the initial value of $u$ and $U$ we adopt the values

$$
\begin{aligned}
u(0) & =\frac{M}{L^{2}}(1+e), \\
a & =U(0)=\frac{3 M^{2}}{L^{2}}(1+e)=b^{2}(1+e),
\end{aligned}
$$

where $e$ is the eccentricity of the orbit. The angular momentum $L^{2}$ can be expressed in terms of the geometric parameters of the orbit by using (21). Thus in physical units we obtain

$$
\begin{aligned}
b^{2} & =\frac{3 G M}{c^{2} \bar{a}\left(1-e^{2}\right)}, \\
a & =\frac{3 G M}{c^{2} \bar{a}(1-e)} .
\end{aligned}
$$

Therefore for this choice of parameters and initial conditions the successive terms in the power series solution of the equation of motion can be obtained by the Laplace-Adomian Decomposition Method as follows:

$$
\begin{aligned}
& u_{0}(\varphi)=\frac{1+e \cos \varphi}{\bar{a}\left(1-e^{2}\right)} \\
& u_{1}(\varphi)=-\frac{G M\left[-3\left(e^{2}+2 e \varphi \sin \varphi+2\right)+e^{2} \cos (2 \varphi)+2\left(e^{2}+3\right) \cos \varphi\right]}{2 c^{2} \bar{a}^{2}\left(1-e^{2}\right)^{2}}, \\
& u_{2}(\varphi)=\frac{G^{2} M^{2}}{16 c^{4} \bar{a}^{3}\left(e^{2}-1\right)^{3}}\left\{e\left[-3 e^{2} \cos (3 \varphi)-16((e-3) e+3) \cos (2 \varphi)+12 \varphi \sin \varphi(8 e \cos \varphi+(4-5 e) e-18)\right]\right. \\
& \left.+\left[e\left((96-29 e) e+72 \varphi^{2}-96\right)+288\right] \times \cos \varphi+48(e((e-3) e+3)+3 \varphi \sin \varphi-6)\right\}, \\
& u_{3}(\varphi)=\frac{G^{3} M^{3}}{16 c^{6} \bar{a}^{4}\left(1-e^{2}\right)^{4}}\left\{e^{4}(-\cos (4 \varphi))+27 e^{3} \varphi \sin (3 \varphi)-24\left[-6 e^{2} \varphi^{2}+4 e(e((e-2) e+6)-6)+3\right] \cos (2 \varphi)\right. \\
& -9(e-3)(e-1) e^{2} \cos (3 \varphi)+\left[-119 e^{4}+348 e^{3}-1125 e^{2}-36(e(e(5 e-2)+18)-6) \varphi^{2}+1152 e-2304\right] \cos \varphi \\
& -3 \varphi\left[e\left(e(e(60 e-209)+348)+24\left(\varphi^{2}-25\right)\right)+504\right] \sin \varphi+12[e((8-5 e) e-42)+24] e \varphi \sin (2 \varphi) \\
& +9[e(e(e(25 e-64)+192)-192)+264]\} \text {, } \\
& u_{4}(\varphi)=\frac{G^{4} M^{4}}{256 c^{8} \bar{a}^{5}\left(e^{2}-1\right)^{5}}\left\{-5 e^{5} \cos (5 \varphi)+192 e^{4} \varphi \sin (4 \varphi)-32(e(2 e-9)+6) \times e^{3} \cos (4 \varphi)\right. \\
& -108[e(e(5 e-12)+66)-36] e^{2} \varphi \sin (3 \varphi)+24 \varphi\left[-580 e^{5}+2038 e^{4}-6177 e^{3}+9522 e^{2}\right. \\
& \left.+12(e(e(15 e-4)+54)-12) \varphi^{2}-12564 e+10224\right] \sin (\varphi)+3\left[-2357 e^{5}+11424 e^{4}-29888 e^{3}+71136 e^{2}\right. \\
& \left.-288 e \varphi^{4}+24(e(e(e(5 e(5 e-24)+569)-600)+1428)-720) \varphi^{2}-73296 e+110592\right] \cos (\varphi) \\
& -192 \varphi\left[e\left(e\left(e(e(20 e-123)+192)+6\left(4 \varphi^{2}-87\right)\right)+396\right)-36\right] \sin (2 \varphi) \\
& -36 e\left[e\left(e\left(e(31 e-72)-54 \varphi^{2}+240\right)-216\right)+36\right] \cos (3 \varphi)-384\left[3 e(e(e(5 e-4)+30)-12) \varphi^{2}\right. \\
& +8 e(e(e(e(2 e-9)+18)-36)+36)-54] \cos (2 \varphi)+288[e(e(e(25 e(2 e-9)+534)-1152)+1152)-1224]\} .
\end{aligned}
$$




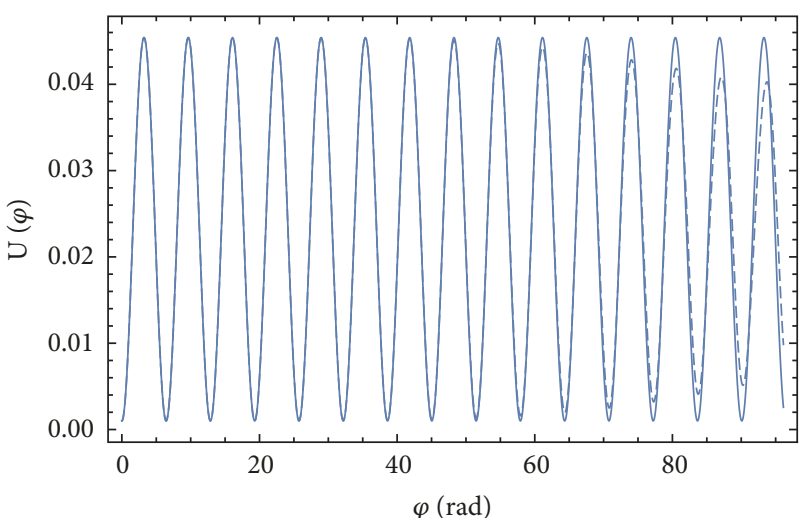

FIGURE 1: Comparison of the numerical solution $U(\varphi)$ of $(80)$ (solid curve) and of the truncated power series solution obtained by the Laplace-Adomian Decomposition Method, given by (105) (dashed curve), for $b^{2}=0.0225$ and $a=0.001$.

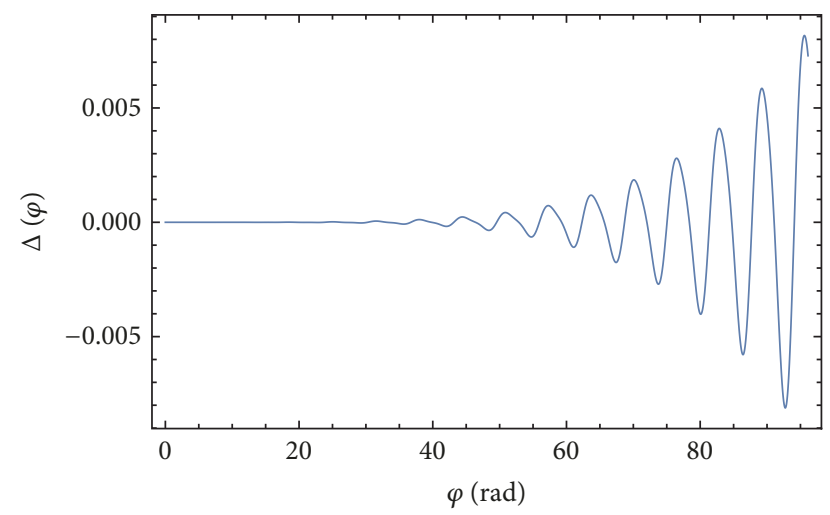

FIgURE 2: The absolute difference $\Delta(\varphi)$ between the numerical solution $\left.U(\varphi)\right|_{\text {num }}$ and the truncated Laplace-Adomian power series solution $\left.U(\varphi)\right|_{\text {LADM }}$ of (80) for $b^{2}=0.0225$ and $a=0.001$.

In the case of the planet Mercury its orbital parameters are $\bar{a}=57.91 \times 10^{11} \mathrm{~cm}$ and $e=0.205615$, respectively. $M$ is the mass of the Sun, given by $M=M_{\odot}=1.989 \times 10^{33} \mathrm{~g}$. Therefore the successive approximations of the solution of the equation of motion of planet Mercury in the Schwarzschild metric created by the gravitational field of the Sun can be obtained as

$$
\begin{aligned}
& u_{0}(\varphi)=1.80305 \times 10^{-13}(1+0.205615 \cos \varphi), \\
& u_{1}(\varphi)=-2.39927 \times 10^{-21}[-3(0.41123 \varphi \sin \varphi \\
& \quad+2.04228)+6.08456 \cos \varphi+0.0422775 \cos (2 \varphi)], \\
& u_{2}(\varphi)=-7.98169 \times 10^{-30}\left\{\left[0.205615\left(72 \varphi^{2}-77.487\right)\right.\right. \\
& \quad+288.00] \cos (\varphi)+48(3 \varphi \sin \varphi-5.50129)
\end{aligned}
$$

$$
\begin{aligned}
& +0.205615[-38.8069 \cos (2 \varphi)-0.126833 \cos (3 \varphi) \\
& +12 \varphi \sin \varphi(1.64492 \cos \varphi-17.3889)]\} \text {, } \\
& u_{3}(\varphi)=2.12421 \times 10^{-37}\left\{-3 \varphi\left[0 . 2 0 5 6 1 5 \left(24\left(\varphi^{2}-25\right)\right.\right.\right. \\
& +63.2396)+504] \sin \varphi+\left(84.2407 \varphi^{2}-2111.88\right) \cos \varphi \\
& -24\left(-0.253665 \varphi^{2}-0.982493\right) \cos (2 \varphi)+38.6365 \varphi \\
& \cdot \sin (2 \varphi)+0.234708 \varphi \sin (3 \varphi)-0.844636 \cos (3 \varphi) \\
& -0.00178739 \cos (4 \varphi)+2089.15\} \text {, } \\
& u_{4}(\varphi)=-3.53332 \times 10^{-46}\left\{24 \varphi\left(7992.95-11.2261 \varphi^{2}\right)\right. \\
& \cdot \sin \varphi \\
& -7.40214\left[0 . 2 0 5 6 1 5 \left(0.205615\left(226.506-54 \varphi^{2}\right)\right.\right. \\
& -216)+36] \cos (3 \varphi) \\
& -192 \varphi\left[0 . 2 0 5 6 1 5 \left(0.205615\left(6\left(4 \varphi^{2}-87\right)+34.4518\right)\right.\right. \\
& +396)-36] \sin (2 \varphi)-384\left(-3.67467 \varphi^{2}-5.82984\right) \\
& \cdot \cos (2 \varphi)+3\left(-59.2171 \varphi^{4}-10728.2 \varphi^{2}+98288.4\right) \\
& \cdot \cos \varphi+104.53 \varphi \sin (3 \varphi)+0.343179 \varphi \sin (4 \varphi) \\
& -1.17779 \cos (4 \varphi)-0.00183757 \cos (5 \varphi)-297094 \text {. } \\
& u(\varphi)=\left(6.88343 \times 10^{-44} \varphi^{3}+8.20724 \times 10^{-36} \varphi\right) \sin (2 \varphi) \\
& +\sin \varphi\left(-3.14475 \times 10^{-36} \varphi^{3}+2.95996 \times 10^{-21} \varphi\right. \\
& \left.-3.23948 \times 10^{-29} \varphi \cos \varphi\right)+\left(1.29321 \times 10^{-36} \varphi^{2}\right. \\
& \left.+5.00886 \times 10^{-36}\right) \times \cos (2 \varphi)+\left(-5.97095 \times 10^{-45} \varphi^{2}\right. \\
& \left.-1.79419 \times 10^{-37}\right) \cos (3 \varphi)+\left(6.27699 \times 10^{-44} \varphi^{4}\right. \\
& \left.-1.18163 \times 10^{-28} \varphi^{2}+3.70733 \times 10^{-14}\right) \cos \varphi \\
& +4.98570 \times 10^{-38} \varphi \sin (3 \varphi)-1.21256 \times 10^{-46} \varphi \\
& \cdot \sin (4 \varphi)-1.01435 \times 10^{-22} \cos (2 \varphi)+2.08152 \\
& \times 10^{-31} \cos (3 \varphi)-3.79680 \times 10^{-40} \cos (4 \varphi)+6.49272 \\
& \times 10^{-49} \cos (5 \varphi)+1.80304 \times 10^{-13} \text {. }
\end{aligned}
$$

4.3.1. The Perihelion Precession. In the first-order approximation the solution of the equation of motion for massive particles in Schwarzschild geometry is obtained as

$$
u(\varphi) \approx u_{0}(\varphi)+u_{1}(\varphi)=\frac{1+e \cos \varphi}{\bar{a}\left(1-e^{2}\right)}+\frac{G M\left[3\left(e^{2}+2 e \phi \sin \varphi+2\right)-e^{2} \cos (2 \varphi)-2\left(e^{2}+3\right) \cos \varphi\right]}{2 \bar{a}^{2} c^{2}\left(1-e^{2}\right)^{2}} .
$$


By neglecting the constant terms and those who oscillate through two cycles on each orbit we obtain

$$
\begin{aligned}
u(\varphi) & \approx \frac{1+e \cos \varphi+e \alpha \varphi \sin \varphi}{l} \\
& =\frac{1+e \cos [(1-\alpha) \varphi]}{l},
\end{aligned}
$$

where we have denoted $l=\bar{a}\left(1-e^{2}\right)$ and $\alpha=3 G M / c^{2} l<<1$. At perihelion we now have $(1-\alpha) \varphi=2 n \pi$, where $n$ is an integer or $\varphi=2 n \pi+6 n \pi\left(G M / l c^{2}\right)$. This relation shows that the perihelion advances by $\Delta \varphi=6 n \pi\left(G M / l c^{2}\right)$ per rotation and the rate of precession is

$$
\frac{\Delta \varphi}{\Delta t}=\frac{6 \pi G M}{\bar{a} c^{2}\left(1-e^{2}\right) \Delta t}, \quad n=1 .
$$

We have thus recovered from the first-order LaplaceAdomian solution of the equation of motion the standard general relativistic result for the perihelion precession [13].

\section{Solving the Equation of Motion for Light in Schwarzschild Geometry}

The equation of motion of light in Schwarzschild geometry is a particular case of the general equation of motion (80) for $b^{2}=0$, and it is given by

$$
\frac{d^{2} U}{d \varphi^{2}}+U=U^{2}
$$

5.1. Power Series Solution. The truncated power series solution of (123) can be obtained immediately by using the Laplace-Adomian Decomposition Method as

$$
\begin{aligned}
& U_{0}(\varphi)=a \cos \varphi \\
& \begin{aligned}
U_{1}(\varphi) & =\frac{2}{3} a^{2} \sin ^{2} \frac{\varphi}{2}(\cos \varphi+2) \\
U_{2}(\varphi) & =\frac{1}{144} a^{3}[60 \varphi \sin \varphi+29 \cos \varphi+16 \cos (2 \varphi)+3 \cos (3 \varphi)-48] \\
U_{3}(\varphi) & =-\frac{1}{432} a^{4}[180 \varphi \sin \varphi+60 \varphi \sin (2 \varphi)+119 \cos \varphi+96 \cos (2 \varphi)+9 \cos (3 \varphi)+\cos (4 \varphi)-225] \\
U_{4}(\varphi) & =\frac{a^{5}}{20736}\left\{\left(7071-1800 \varphi^{2}\right) \cos \varphi+6144 \cos (2 \varphi)+1116 \cos (3 \varphi)+64 \cos (4 \varphi)+5[12(232 \varphi \sin \varphi\right. \\
+64 \varphi \sin (2 \varphi)+9 \varphi \sin (3 \varphi)-240)+\cos (5 \varphi)]\}, & \\
U(\varphi) & =\frac{a}{20736}\left\{a \left[384\left(2 a\left(8 a^{2}-6 a+3\right)-9\right) \cos (2 \varphi)+a(36(a(31 a-12)+12) \times \cos (3 \varphi)\right.\right. \\
+a & (16(4 a-3) \cos (4 \varphi)+5 a \cos (5 \varphi)+60 \varphi \sin \varphi(18 a \cos (2 \varphi)+32(4 a-3) \cos \varphi+241 a-144))+8640 \varphi \sin \varphi) \\
- & \left.144(a(25 a(4 a-3)+48)-72)]+3\left[a\left(a\left(a\left(a\left(2357-600 \varphi^{2}\right)-1904\right)+1392\right)-2304\right)+6912\right] \cos \varphi\right\}
\end{aligned}
\end{aligned}
$$

The comparison of the numerical solution of (123) and its truncated power series solution (129), as well as their difference, is presented in Figures 3 and 4, respectively.

5.2. The Bending Angle of Light. In the Newtonian approximation the solution of the equation of motion for the light has the solution $u_{0}=\cos \varphi / R$, where $R$ is the distance of the closest approach to the massive object. This fixes the value of the constant $a$ as $a=3 G M / c^{2} R$. In the first order of approximation the equation of motion of the photon in the Schwarzschild geometry has the solution

$$
\begin{aligned}
u(\varphi) & \approx u_{0}(\varphi)+u_{1}(\varphi) \\
& =\frac{1}{R} \cos \varphi+\frac{2 G M}{c^{2} R^{2}} \sin ^{2} \frac{\varphi}{2}(2+\cos \varphi) .
\end{aligned}
$$

By taking $\varphi=\pi / 2+\epsilon$, we have

$$
\begin{aligned}
& u(\varphi) \\
& \quad \approx \frac{G M}{2 c^{2} R^{2}}\left\{2\left(1-\frac{c^{2} R}{G M}\right) \sin \epsilon+[\cos (2 \epsilon)+3]\right\} .
\end{aligned}
$$

By performing a first-order series expansion with respect to $\epsilon$ and taking $u=0$, it gives the light deflection angle as

$$
\delta=2 \epsilon \approx \frac{4 G M}{c^{2} R\left(1-G M / c^{2} R\right)},
$$

which in the limit $G M / c^{2} R<<1$ reduces to the well-known general relativistic result $\delta \approx 4 G M / c^{2} R$ [13]. In the second 


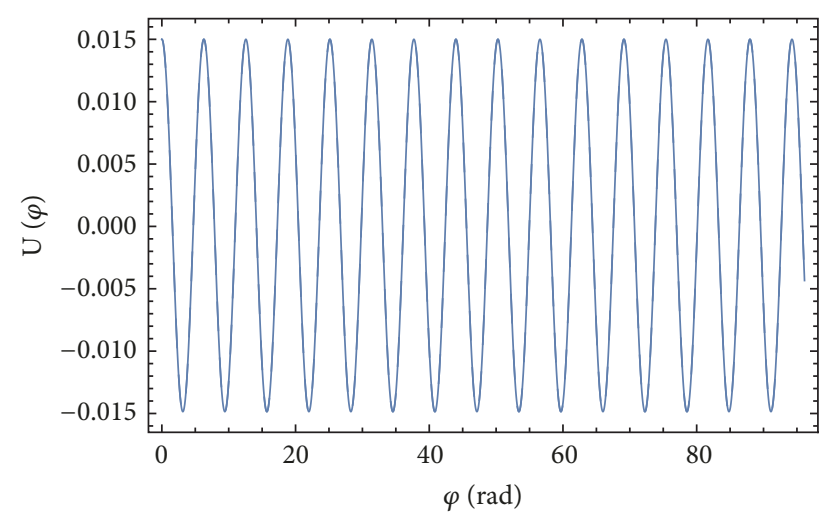

FIgURE 3: Comparison of the numerical solution $U(\varphi)$ of (123), describing the motion of a photon in Schwarzschild geometry (solid curve), and of its truncated power series solution obtained by the Laplace-Adomian Decomposition Method, given by (129) (dashed curve), for $a=0.015$.

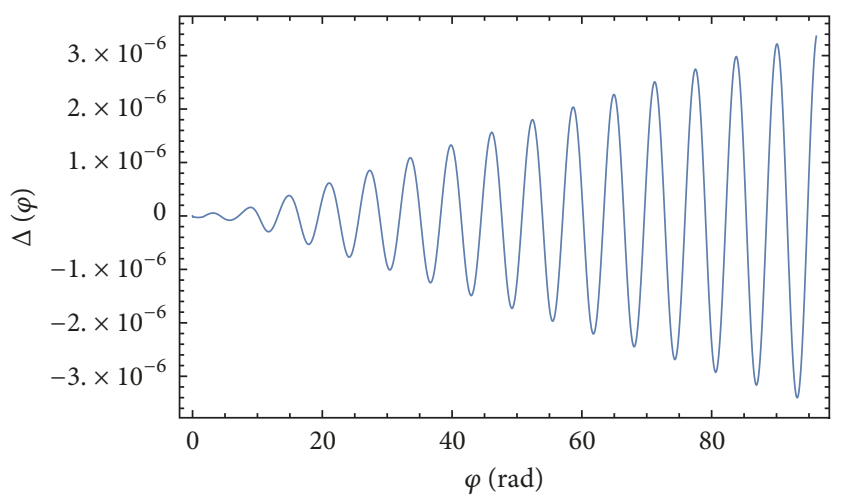

Figure 4: The absolute difference $\Delta(\varphi)$ between the numerical solution $\left.U(\varphi)\right|_{\text {num }}$ and the truncated Laplace-Adomian power series solution $\left.U(\varphi)\right|_{\text {LADM }}$ of Eq. (123) for $a=0.015$.

order of approximation, with $u \approx u_{0}+u_{1}+u_{2}$, we obtain by using the same procedure

$$
\delta=\frac{4 G M}{c^{2} R} \frac{1+(15 \pi / 16-2)\left(G M / c^{2} R\right)}{1-G M / c^{2} R-5 G^{2} M^{2} / 2 c^{4} R^{2}} .
$$

Finally, in the fourth order of approximation we obtain

$$
\begin{aligned}
\delta \approx & \frac{4 G M}{c^{2} R}\left(1+\frac{(15 \pi-32)}{16} \frac{G M}{c^{2} R}\right. \\
& \left.-\frac{5(9 \pi-32)}{16} \frac{G^{2} M^{2}}{c^{4} R^{2}}+\frac{5(669 \pi-2048)}{256} \frac{G^{3} M^{3}}{c^{6} R^{3}}\right) \\
& \cdot\left(1-\frac{G M}{c^{2} R}-\frac{5 G^{2} M^{2}}{2 c^{4} R^{2}}-\frac{(15 \pi-22)}{4} \frac{G^{3} M^{3}}{c^{6} R^{3}}\right. \\
& \left.-\frac{\left(4816-1920 \pi+225 \pi^{2}\right)}{128} \frac{G^{4} M^{4}}{c^{8} R^{4}}\right)^{-1} .
\end{aligned}
$$

\section{Discussions and Concluding Remarks}

In the present paper we have considered the applications of a very powerful mathematical method, the LaplaceAdomian Decomposition Method, for the study of the general relativistic equations describing the motions of massive test particles and of photons in spherical symmetric geometries. The Laplace-Adomian Decomposition Method has been used extensively for obtaining power series solutions of many classes of nonlinear differential and integral equations. High precision solutions can be obtained by using only a few terms in a truncated series expansion. The main "ingredients" in this method are the Adomian polynomials, which are generated recursively in a step by step procedure.

After introducing a general formalism for obtaining the equations of motion in arbitrary spherically symmetric spacetimes and after the presentation of the LaplaceAdomian Decomposition Method, we have investigated in detail the dynamics of massive particles and photons in Schwarzschild geometry. The equations of motion can be easily solved, and we have obtained their series solution. One of the advantages of the Laplace-Adomian Decomposition Method is that it gives directly the solution in terms of trigonometric functions, and not in terms of power series of the angular variable $\varphi$. A truncated series solution containing only five terms can reproduce the exact numerical solution with a high precision. Moreover, obtaining the terms in the Adomian series can be easily done with the use of some symbolic software. The basic known physical results can be easily reobtained in the first-order approximation, as well as for higher orders. For the perihelion precession we have investigated only the first-order effects, but in the case of the deflection of light we have obtained the bending angle up to the fourth order of approximation.

Several important generalizations and extensions of the Schwarzschild metric are known. In the case of the vacuum spacetimes outside charged spherically symmetric objects carrying a charge $Q$ the geometry is given by the ReissnerNordstrom metric,

$$
e^{\nu}=e^{-\lambda}=1-\frac{2 G M}{c^{2} r}+\frac{Q^{2}}{r^{2}} .
$$

In the case of rotating star the first-order monopole correction to the Schwarzschild metric is given by [49]

$$
e^{\nu}=e^{-\lambda}=1-\frac{2 G M}{c^{2} r}+\frac{J^{2}}{r^{4}},
$$

where $J=I \Omega$, where $I$ is the moment of inertia, $\Omega$ is the angular velocity, and $J$ is the angular momentum of the star, respectively.

The physics of the particle motion in these metrics can be easily investigated by using the methods developed in the present paper. For example, in the case of the ReissnerNordstrom metric, $f(u)=2 G M u / c^{2}-Q u^{2}$,

$$
\begin{aligned}
G(u)= & 2 \frac{G M}{c^{2}} u^{3}-Q u^{4}+\frac{E^{2}}{c^{2} L^{2}}-\frac{1}{L^{2}}+\frac{2 G M u}{c^{2} L^{2}} \\
& -\frac{Q u^{2}}{L^{2}},
\end{aligned}
$$


and

$$
F(u)=\frac{3 G M}{c^{2}} u^{2}-2 Q u^{3}+\frac{G M}{c^{2} L^{2}}-\frac{Q u}{L^{2}},
$$

respectively. Therefore the equation of motion in the Reissner-Nordstrom metric is given by

$$
\frac{d^{2} u}{d \varphi^{2}}+\left(1+\frac{Q}{L^{2}}\right) u=\frac{3 G M}{c^{2}} u^{2}-2 Q u^{3}+\frac{G M}{c^{2} L^{2}},
$$

which can be solved easily by using the Laplace-Adomian Decomposition Method. The radius of the circular orbit $u_{0}$ can be obtained as a solution of the algebraic equation

$$
\frac{3 G M}{c^{2}} u_{0}^{2}-u_{0}+\frac{G M}{c^{2} L^{2}}=2 Q u_{0}^{3}+\frac{Q}{L^{2}} u_{0} .
$$

In the first order of approximation and with the assumption $\mathrm{Q} / L^{2} \ll 1, u_{0}$ can be approximated as $u_{0} \approx G M / c^{2} L^{2}$. Thus, the perihelion precession $\Delta \phi$ in the Reissner-Nordstrom geometry is given by

$$
\Delta \phi=\frac{6 \pi G M}{c^{2} a\left(1-e^{2}\right)}-\frac{\pi c^{2} Q}{G M a\left(1-e^{2}\right)} .
$$

The first term in (141) gives the general relativistic correction term for the perihelion precession, while the second term gives the correction due to the presence of the charge. In the case of the motion of massless particles we have

$$
P(u)=2 \frac{G M}{c^{2}} u^{3}-Q u^{4}+\frac{E^{2}}{c^{2} L^{2}},
$$

and

$$
V(u)=\frac{3 G M}{c^{2}} u^{2}-2 Q u^{3} .
$$

Hence the equation of motion for photons is given by

$$
\frac{d^{2} u}{d \phi^{2}}+u=\frac{3 G M}{c^{2}} u^{2}-2 Q u^{3}
$$

This equation can also be investigated easily by using the Laplace-Adomian Decomposition Method, and the methods are developed in the present paper.

\section{Data Availability}

There are no underlying data for this manuscript.

\section{Conflicts of Interest}

The authors declare that they have no conflicts of interest.

\section{References}

[1] K.-H. Lo, K. Young, and B. Y. P. Lee, "Advance of perihelion," American Journal of Physics, vol. 81, pp. 695-702, 2013.

[2] R. S. Park, W. M. Folkner, A. Konopliv, J. G. Williams, D. E. Smith, and M. T. Zuber, "Precession of Mercury's Perihelion from Ranging to the MESSENGER Spacecraft," The Astronomical Journal, vol. 153, p. 121, 2017.
[3] D. E. Lebach, B. E. Corey, I. I. Shapiro et al., "Measurement of the Solar Gravitational Deflection of Radio Waves Using VeryLong-Baseline Interferometry," Physical Review Letters, vol. 75, no. 8, pp. 1439-1442, 1995.

[4] O. Titov and A. Girdiuk, "Recent developments and prospects in ground-based and space astrometry, held at Pulkovo Observatory," in Proceedings of the Proceedings of the Journés 2014, Z. Malkin and N. Capitaine, Eds., pp. 75-78, 2014.

[5] S. S. Shapiro et al., "Measurement of the Solar Gravitational Deflection of Radio Waves using Geodetic Very-Long-Baseline Interferometry Data, 1979-1999," Physical Review Letters, vol. 92, Article ID 121101, 2004.

[6] E. Fomalont, S. Kopeikin, G. Lanyi, and J. Benson, "Progress in measurements of the gravitational bending of radio waves using the vlba," The Astrophysical Journal , vol. 699, no. 2, pp. 13951402, 2009.

[7] F. Zhang, Y. Lu, and Q. Yu, "on Testing The Kerr Metric of The Massive Black Hole in The Galactic Center via Stellar Orbital Motion: Full General Relativistic Treatment," The Astrophysical Journal, vol. 809, p. 127, 2015.

[8] Q. Yu, F. Zhang, and Y. Lu, "Prospects for Constraining The Spin of The Massive Black Hole at The Galactic Center via The Relativistic Motion of A Surrounding Star," The Astrophysical Journal, vol. 827, p. 114, 2016.

[9] A. Hees, T. Do, A. M. Ghez et al., "Testing General Relativity with Stellar Orbits around the Supermassive Black Hole in Our Galactic Center," Physical Review Letters, vol. 118, Article ID 211101, 2017.

[10] M. Parsa, "Investigating the Relativistic Motion of the Stars Near the Supermassive Black Hole in the Galactic Center," The Astrophysical Journal, vol. 845, p. 22, 2017.

[11] C. M. Will, "The confrontation between general relativity and experiment," Living Reviews in Relativity, vol. 17, no. 4, 2014.

[12] W.-T. Ni, "Equivalence principles, spacetime structure and the cosmic connection," International Journal of Modern Physics D: Gravitation, Astrophysics, Cosmology, vol. 25, no. 4, 1630002, 51 pages, 2016.

[13] L. D. Landau and E. M. Lifshitz, The Classical Theory of Fields, Pergamon Press, Oxford, UK, 1951.

[14] G. V. Kraniotis and S. B. Whitehouse, "Compact calculation of the perihelion precession of Mercury in general relativity, the cosmological constant and Jacobi's inversion problem," Classical and Quantum Gravity, vol. 20, no. 22, pp. 4817-4835, 2003.

[15] Y.-P. Hu, H. Zhang, J.-P. Hou, and L.-Z. Tang, "Perihelion Precession and Deflection of Light in the General Spherically Symmetric Spacetime," Advances in High Energy Physics, vol. 2014, Article ID 604321, 7 pages, 2014.

[16] J.-H. He, "Homotopy perturbation method: a new nonlinear analytical technique," Applied Mathematics and Computation, vol. 135, no. 1, pp. 73-79, 2003.

[17] V. K. Shchigolev, "Analytical computation of the perihelion precession in general relativity via the homotopy perturbation method," Universal Journal of Computational Mathematics, vol. 3, no. 4, pp. 45-49, 2015.

[18] V. Shchigolev and D. Bezbatko, "On HPM approximation for the perihelion preces-sion angle in general relativity," International Journal of Advanced Astronomy, vol. 5, no. 1, p. 38, 2017.

[19] G. Adomian, "A review of the decomposition method in applied mathematics," Journal of Mathematical Analysis and Applications, vol. 135, no. 2, pp. 501-544, 1988. 
[20] G. Adomian, Solving Frontier Problems of Physics: The Decomposition Method, Kluwer Academic, Dordrecht, The Netherlands, 1994.

[21] G. Adomian and R. Rach, "Modified Adomian polynomials," Mathematical and Computer Modelling, vol. 24, no. 11, pp. 3946, 1996.

[22] Y. Cherruault, G. Adomian, K. Abbaoui, and R. Rach, "Further remarks on convergence of decomposition method," International Journal of Bio-Medical Computing, vol. 38, no. 1, pp. 8993, 1995.

[23] G. Adomian, "Decomposition solution for Duffing and van der Pol oscillators," International Journal of Mathematics and Mathematical Sciences, vol. 9, no. 4, pp. 731-732, 1986.

[24] G. Adomian, "On the convergence region for decomposition solutions," Journal of Computational and Applied Mathematics, vol. 11, no. 2, pp. 379-380, 1984.

[25] G. Adomian, "On the convergence region for decomposition solutions," Journal of Computational and Applied Mathematics, vol. 11, no. 27, pp. 379-380, 1984.

[26] A.-M. Wazwaz, "The modified decomposition method and Padé approximants for solving the Thomas-Fermi equation," Applied Mathematics and Computation, vol. 105, no. 1, pp. 11-19, 1999.

[27] A.-M. Wazwaz, "A reliable modification of Adomian decomposition method," Applied Mathematics and Computation, vol. 102, no. 1, pp. 77-86, 1999.

[28] A.-M. Wazwaz, "Adomian decomposition method for a reliable treatment of the Emden-Fowler equation," Applied Mathematics and Computation, vol. 161, no. 2, pp. 543-560, 2005.

[29] X.-G. Luo, "A two-step Adomian decomposition method," Applied Mathematics and Computation, vol. 170, no. 1, pp. 570583, 2005.

[30] B.-Q. Zhang, Q.-B. Wu, and X.-G. Luo, "Experimentation with two-step Adomian decomposition method to solve evolution models," Applied Mathematics and Computation, vol. 175, no. 2, pp. 1495-1502, 2006.

[31] E. Babolian and S. Javadi, "Restarted Adomian method for algebraic equations," Applied Mathematics and Computation, vol. 146, no. 2-3, pp. 533-541, 2003.

[32] E. Babolian, S. Javadi, and H. Sadeghi, "Restarted Adomian method for integral equations," Applied Mathematics and Computation, vol. 153, no. 2, pp. 353-359, 2004.

[33] C. Jin and M. Liu, "A new modification of Adomian decomposition method for solving a kind of evolution equation," Applied Mathematics and Computation, vol. 169, no. 2, pp. 953-962, 2005.

[34] H. Jafari and V. Daftardar-Gejji, "Revised Adomian decomposition method for solving a system of nonlinear equations," Applied Mathematics and Computation, vol. 175, no. 1, pp. 1-7, 2006.

[35] H. Jafari and V. Daftardar-Gejji, "Revised Adomian decomposition method for solving systems of ordinary and fractional differential equations," Applied Mathematics and Computation, vol. 181, no. 1, pp. 598-608, 2006.

[36] R. Rach, G. Adomian, and R. E. Meyers, Computers \& Mathematics with Applications, vol. 23, p. 17, 1992.

[37] A.-M. Wazwaz and S. M. El-Sayed, "A new modification of the Adomian decomposition method for linear and nonlinear operators," Applied Mathematics and Computation, vol. 122, no. 3, pp. 393-405, 2001.
[38] H. O. Bakodah, "Some modifications of Adomian decomposition method applied to nonlinear system of Fredholm integral equations of the second kind," International Journal of Contemporary Mathematical Sciences, vol. 7, no. 17-20, pp. 929942, 2012.

[39] J. Biazar, E. Babolian, and R. Islam, "Solution of the system of ordinary differential equations by Adomian decomposition method," Applied Mathematics and Computation, vol. 147, no. 3, pp. 713-719, 2004.

[40] J. Biazar, E. Babolian, G. Kember, A. Nouri, and R. Islam, "An alternate algorithm for computing Adomian polynomials in special cases," Applied Mathematics and Computation, vol. 138, no. 2-3, pp. 523-529, 2003.

[41] P.-Y. Tsai and C.-K. Chen, "Free vibration of the nonlinear pendulum using hybrid Laplace Adomian decomposition method," International Journal for Numerical Methods in Biomedical Engineering, vol. 27, no. 2, pp. 262-272, 2011.

[42] M. Azreg-Ainou, "Developed Adomian method for quadratic Kaluza-KLEin relativity," Classical and Quantum Gravity, vol. 27, no. 1, Article ID 015012, 16 pages, 2010.

[43] G. Esposito, E. Battista, and E. Di Grezia, "Bicharacteristics and Fourier integral operators in Kasner spacetime," International Journal of Geometric Methods in Modern Physics, vol. 12, no. 5, 1550060, 29 pages, 2015.

[44] C. G. Boehmer, T. Harko, and F. S. Lobo, "Solar system tests of brane world models," Classical and Quantum Gravity, vol. 25, no. 4, Article ID 045015, 12 pages, 2008.

[45] T. Harko, Z. Kovács, F. S. N. Lobo, and R. Soc, "Solar System tests of Hořava-Lifshitz gravity," Proceedings of the Royal Society A Mathematical, Physical and Engineering Sciences, vol. 467, no. 2129, pp. 1390-1407, 2011.

[46] C. G. Boehmer, G. De Risi, T. Harko, and F. S. N. Lobo, "Classical tests of general relativity in brane world models," Classical and Quantum Gravity, vol. 27, no. 18, Article ID 185013, 2010.

[47] G. D. Risi, T. Harko, and F. S. Lobo, "Solar System constraints on local dark matter density," Journal of Cosmology and Astroparticle Physics, vol. 2012, no. 7, 47 pages, 2012.

[48] L. D. Landau and E. M. Lifshitz, Mechanics, ButterworthHeinemann, Oxford, UK, 2000.

[49] N. K. Glendenning and F. Weber, "Impact of frame dragging on the Kepler frequency of relativistic stars," Physical Review D: Particles, Fields, Gravitation and Cosmology, vol. 50, no. 6, pp. 3836-3841, 1994. 

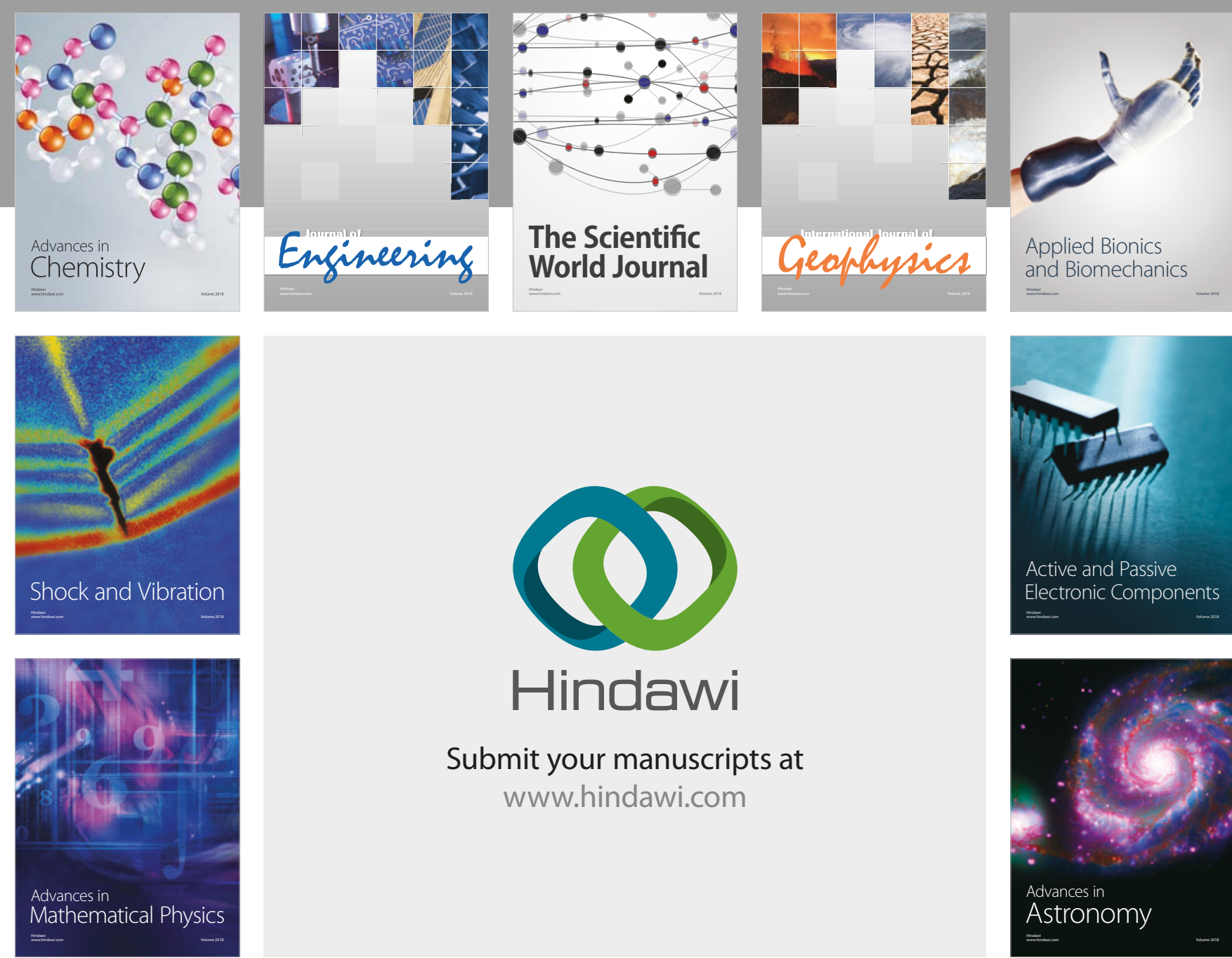

Submit your manuscripts at

www.hindawi.com

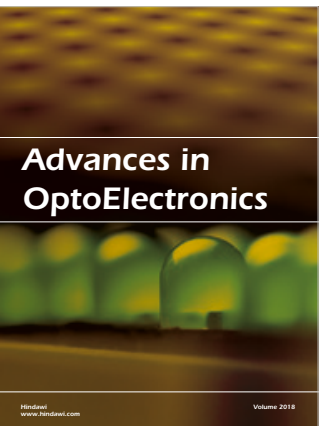

\section{Rotcting Machinery}
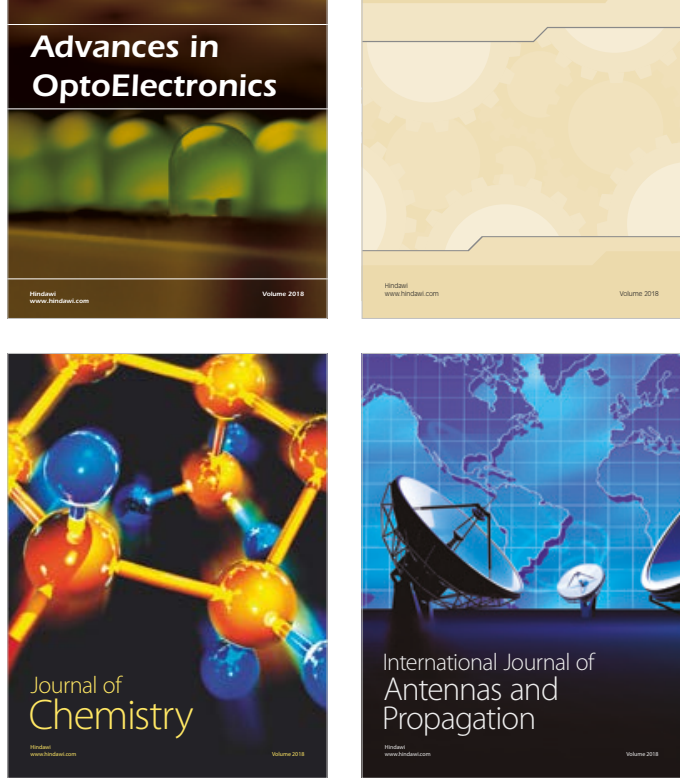

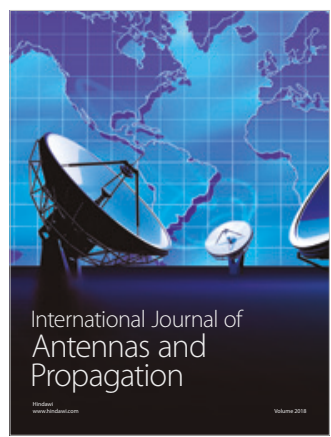

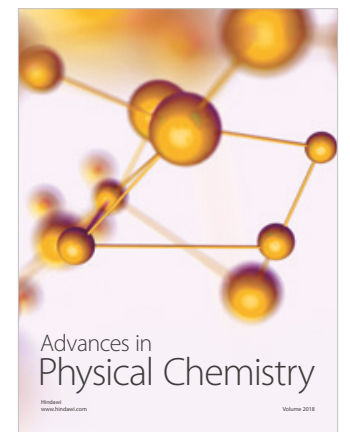

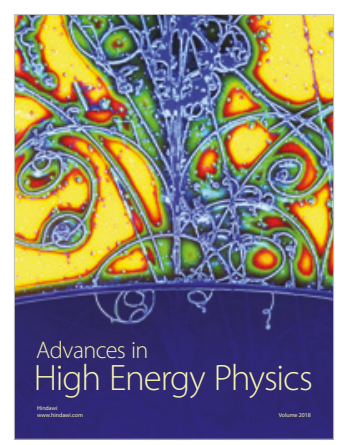

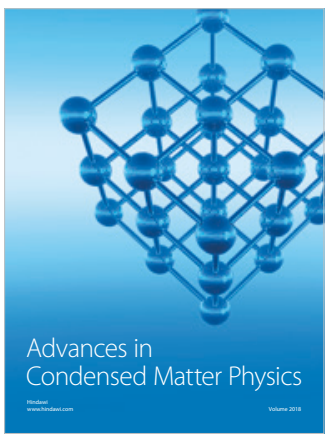

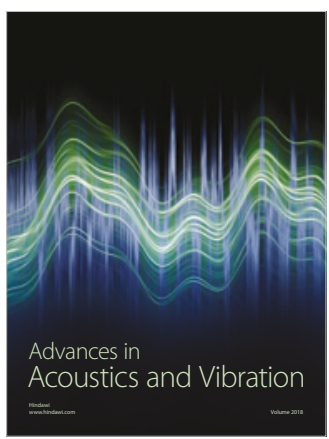

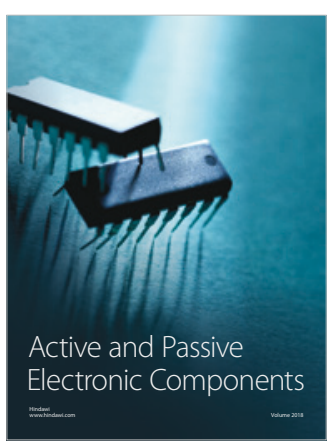
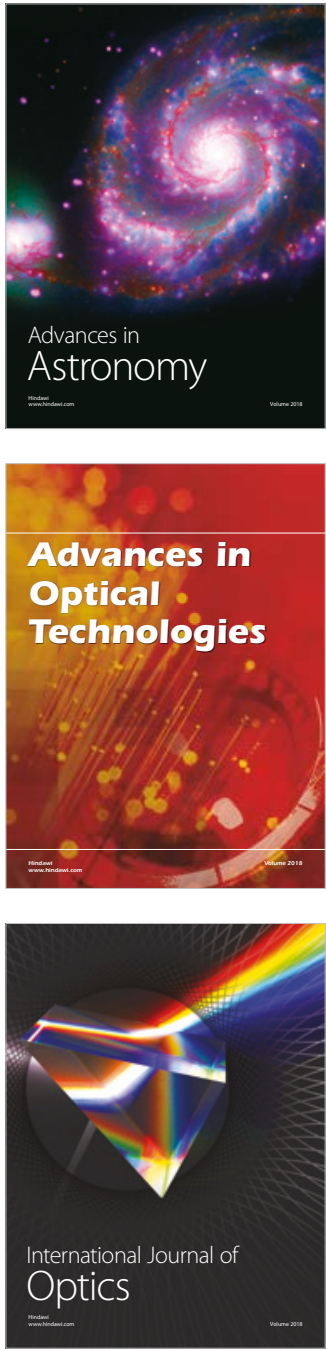